\title{
Bringt Budgetmedizin doch keine Einsparungen?
}

Am 17. Juni wird an der Urne entschieden, ob die freie Arztwahl der Budgetmedizin geopfert wird. Wer sich nicht in einem Budgetnetzwerk versichert, soll im Krankheitsfall mehr bezahlen. Legitimiert wird dies mit Kosteneffizienz, für die es allerdings bis heute keine Evidenz gibt. Auch die kürzlich in der SÄZ publizierte ökonomische Studie ändert nichts daran.

René Haldemann

Korrespondenz:

Dr. med. René Haldemann Gartenstrasse 8

CH-8805 Richterswil

Tel. 0438889575

Fax 0438889576

rene.haldemann[at]hin.ch
Unter Managed Care (MC) wird jede Form von Steuerung von Gesundheitsleistungen unter dem betriebswirtschaftlichen Aspekt verstanden. Bereits das heutige Krankenversicherungsgesetz (KVG) erlaubt Versicherungsformen, bei denen für einen Prämienrabatt auf die freie Arztwahl verzichtet werden kann. Hierzu zählen Hausarztmodelle, die telefonische Beratung vor dem Arztbesuch und HMOs mit oder ohne Budgetverantwortung. Wettbewerbsverzerrend begünstigt die Reform integrierende Versorgungsnetzwerke mit Budgetmitverantwortung, während die anderen MC-Produkte leer ausgehen. Wer sich nicht einem solchen Budgetnetzwerk anschliesst, wird im Krankheitsfall mit einem Selbstbehalt von $15 \%$ statt 10\% gebüsst. Die Befürworter der Reform proklamieren Kosteneinsparungen ohne Abstriche bei der Behandlungsqualität. Unter dem Titel «Steigern die Schweizer Ärztenetzwerke die Effizienz im Gesundheitswesen?» wurde kürzlich eine Studie [1, 2] publiziert, die sogar mediale Aufmerksamkeit erlangte. Die Protagonisten der Budgetmedizin fühlen sich aufgrund der Resultate bestätigt. Der vorliegende Artikel nimmt die Ergebnisse auf dem Hintergrund der KVG-Reform kritisch unter die Lupe.

\section{Details zur Methodik der Studie}

Die Studie wurde von dem CSS-Institut durchgeführt, das vom gleichnamigen Krankenversicherer gesponsert wird. Retrospektiv wurden die in der obligatorischen Krankenpflegeversicherung (OKP) anfallenden Behandlungskosten bei einer ärztlichen Versorgung im Budgetnetzwerk und bei einer traditionellen Versicherungsform mit freier Arztwahl miteinander verglichen. Es wurden sechs verschiedene Netzwerke analysiert und die Ergebnisse anschliessend über mehrere Jahre (2006-2009) gepoolt, womit es sich formal um eine Metaanalyse handelt. Da nur der Aufwand in Form von Kosten aus der Perspektive des Krankenversicherers analysiert wurde und auf der Ergebnisseite keine Nutzwerte (Behandlungsergebnis) erhoben wurden, handelt es sich um eine reine Kostenvergleichstudie. Sodann sind Aussagen zur Kosteneffizienz im gesundheitsökonomischen Sinne nicht zulässig.

\section{Studienkollektive}

Bei allen sechs Ärztenetzwerken sind die Versichertenkollektive jünger und gesünder, da ältere und kränkere Versicherte sich eher für eine normale hausärztliche Versorgung mit freier Arztwahl entscheiden. Damit ein Kostenvergleich unter diesen Bedingungen überhaupt möglich ist, wurden mathematische Verfahren appliziert, welche diese Morbiditätsunterschiede künstlich ausgleichen. So wurden aus dem Pool der Normalversicherten Kollektive gebildet, die in Bezug auf eine Reihe von kostenrelevanten Risikomerkmalen mit der Versichertenpopulation im Netzwerk vergleichbar sind. Da die Fall-Kontrollanalyse (Zwillingsanalyse) und die CART-Methode mehr Risikomerkmale als die BAGMethode berücksichtigen, fallen die Einsparungen geringer aus. Auch wenn uns in der Originalarbeit die gepoolten Werte für die Risikomerkmale vorenthalten werden, lässt sich aus den tabellarischen Daten der Einzelstudien für die Zwillingsanalyse rasch erkennen, dass die Normalversicherten im Vergleich zum Netzwerkkollektiv etwa 4-6 Jahre älter sind, der Anteil in einer kostenrelevanten Pharmaceutical Cost Group (PCG) um einen Faktor 1,5-2,5 Mal höher ist, eine Multimorbidität 2-4 Mal häufiger vorliegt, und der Anteil der Versicherten, die im Vorjahr hospitalisiert waren, auch 2-2,5 Mal so oft vorkommt. Somit ist der Spareffekt durch die Risikoselektion ein Vielfaches höher als die berechnete risikobereinigte Einsparung.

\section{«Die Risikoselektion erklärt einen Grossteil der Bruttoeinsparungen.»}

\section{Ergebnisse}

Die Autoren kommen zum Schluss, dass ihre Forschungsarbeit deutlich zeigt, dass die Schweizer Ärztenetzwerke auch auf mittlere Frist erhebliche Einsparungen erzielen und so zur Effizienzsteigerung im Schweizer Gesundheitswesen beitragen. Solche Aus- 


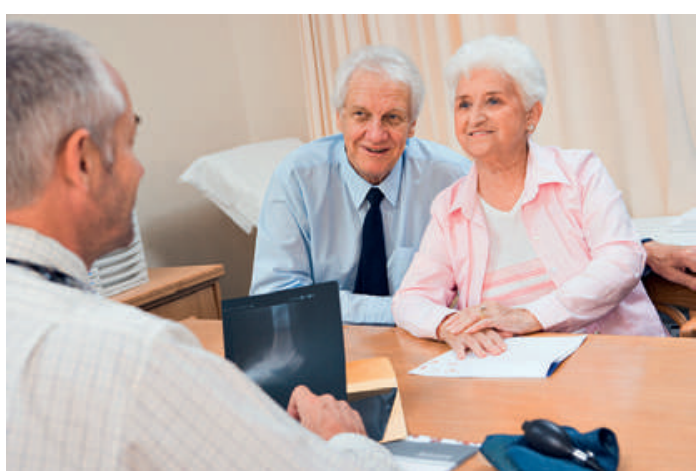

Die Demographie und der medizinische Fortschritt sind Kostentreiber, denen sich auch die Budgetmedizin nicht entziehen kann.

sagen sind Balsam für die Seele der verzweifelten Budgetmediziner, die nun glauben, dass der langersehnte Beweis für den Kostenvorteil endlich erbracht wurde. Mit den konservativen Berechnungsmethoden (Zwillings- und CART-Analyse) wurden mittlere Einsparungen von $11,9 \%$ bis $13,0 \%$ ermittelt, wobei erhebliche Unterschiede zwischen den Netzwerken vorliegen. So werden für die zuvor gennannten Mit-

\section{Für die Effizienzsteigerungen in der hausärztlichen Versorgung braucht es gesunden Wettbewerb, keine staatlichen Interventionen.}

telwerte Standardabweichung von $8,6 \%$ und $6,7 \%$ angegeben. Das Mass der Streuung wird mit dem 95\% Konfidenzintervall ausgedrückt, das von der Grösse der Studienpopulation und der Standardabweichung abhängt. In jedem Fall beträgt es aber mindestens das 1,96fache der Standardabweichung, sodass die Einsparungen statistisch gesehen nicht mehr signifikant sind. Es ist nicht einzusehen, weshalb für Netzwerkmedizin mit Budgetverantwortung andere statistische Massstäbe gelten als für andere medizinische Interventionen.

Die risikobereinigten Einsparungen für die drei Methoden wurden für die einzelnen Netzwerke graphisch dargestellt. Bei deren Betrachtung entsteht der Eindruck, dass die Einsparungen über die Jahre abnehmen. Dieser Trend könnte eine Regression zur Mitte bedeuten, weil sich die Kollektive bezüglich der Krankheitslast mittel- bis langfristig immer mehr annähern und die Berechnungsmethoden somit auch weniger fehleranfällig sind. Es wäre aufschlussreich gewesen, wenn die risikobereinigten Einsparungen gepoolt und pro Jahr mit den zugehörigen Konfidenzintervallen dargestellt und ein Trend mit statistischen Methoden überprüft worden wäre.

\section{Schlussfolgerungen}

Die Risikoselektion erklärt einen Grossteil der Bruttoeinsparungen. Die verbleibenden risikobereinigten Kosteneinsparungen sind statistisch nicht signifikant. Solange die Budgetmedizin nicht rationiert, ist der kostenbewusste Hausarzt also nicht teurer als der Netzwerkdoktor. So wäre es nicht weiter verwunderlich, wenn die Einsparungen über den Beobachtungszeitraum tatsächlich abnehmen. Nach den Vorstellungen des Bundesrates und des Parlamentes sollen $60 \%$ der Versicherten durch die planwirtschaftliche Reform in die Netzwerke gezwungen werden. Dies führt dazu, dass sich die Morbiditätslasten der Netzwerkversicherten und der Normalversicherten angleichen, sodass sich ohne Rationierung von medizinischen Leistungen die Einsparungen sehr wahrscheinlich in Luft auflösen würden.

\section{Diskussion}

Die Hausarztmedizin kann auch ohne budgetären Druck kostenbewusst sein und den Patienten ohne falsche Anreize beraten. Für die Effizienzsteigerungen in der hausärztlichen Versorgung braucht es einen gesunden Wettbewerb und keine staatlichen Interventionen, die ein einziges Versicherungsmodell finanziell begünstigt, von dem wir nicht einmal wissen, ob es die Vorzugsbehandlung beim differenzierten Selbstbehalt überhaupt verdient. Die diskutierte Studie liefert auf jeden Fall keine Legitimation hierfür. Die Demographie und der medizinische Fortschritt sind Kostentreiber, denen sich auch die Budgetmedizin nicht entziehen kann. Prozessoptimierungen brauchen kein Budget, sondern selbstbewusste Hausärzte. Budgetmedizin beschädigt das Vertrauensverhältnis zum Patienten, der sich nie ganz sicher ist, ob die Vorenthaltung einer Leistung nicht doch budgetär motiviert ist. Die Politiker bürden den Hausärzten in den Budgetnetzwerken geschickt die Rolle des Rationierers auf. Die steigenden Gesundheitskosten machen Rationierung tatsächlich unumgänglich. In diesem Punkt müssen wir Ärzte nolens volens kooperieren. Wird das Referendum angenommen, ist der Weg frei für neue Lösungsansätze, bei denen wir Ärzte uns allerdings entschlossener und geeinter als bisher einbringen müssen.

\section{Literatur}

1 Trottmann D, Beck K, Kunze U. Steigern Schweizer Ärztenetzwerke die Effizienz im Gesundheitswesen? Schweiz Ärztezeitung. 2012;93(4):125-7.

2 Beck K, Kunze U, Buholzer M, Trottmann M. Steigern Schweizer Ärztenetzwerke die Effizienz im Gesundheitswesen? Eine Analyse auf mittlere Frist. Forschungsbericht des CSS Institut für Empirische Gesundheitsökonomie; 2011 (www.css-institut.ch). 